\title{
Rev1, Rev3, or Rev7 siRNA Abolishes Ultraviolet Light-Induced Translesion Replication in HeLa Cells: A Comprehensive Study Using Alkaline Sucrose Density Gradient Sedimentation
}

\author{
Jun Takezawa, ${ }^{1}$ Yukio Ishimi, ${ }^{2}$ Naomi Aiba, ${ }^{1}$ and Kouichi Yamada ${ }^{1}$ \\ ${ }^{1}$ Division of Genetic Biochemistry, The National Institute of Health and Nutrition, Shinjuku-ku, Tokyo 162-8636, Japan \\ ${ }^{2}$ Faculty of Science, Ibaraki University, Mito-shi, Ibaraki-ken 310-8512, Japan \\ Correspondence should be addressed to Kouichi Yamada, kouichi@nih.go.jp
}

Received 14 May 2010; Revised 13 July 2010; Accepted 17 September 2010

Academic Editor: Shigenori Iwai

Copyright () 2010 Jun Takezawa et al. This is an open access article distributed under the Creative Commons Attribution License, which permits unrestricted use, distribution, and reproduction in any medium, provided the original work is properly cited.

When a replicative DNA polymerase stalls upon encountering a lesion on the template strand, it is relieved by other lowprocessivity polymerase(s), which insert nucleotide(s) opposite the lesion, extend by a few nucleotides, and dissociate from the $3^{\prime}-\mathrm{OH}$. The replicative polymerase then resumes DNA synthesis. This process, termed translesion replication (TLS) or replicative bypass, may involve at least five different polymerases in mammals, although the participating polymerases and their roles have not been entirely characterized. Using siRNAs originally designed and an alkaline sucrose density gradient sedimentation technique, we verified the involvement of several polymerases in ultraviolet (UV) light-induced TLS in HeLa cells. First, siRNAs to Rev3 or Rev7 largely abolished UV-TLS, suggesting that these 2 gene products, which comprise Pol $\zeta$, play a main role in mutagenic TLS. Second, Rev1-targeted siRNA also abrogated UV-TLS, indicating that Rev1 is also indispensable to mutagenic TLS. Third, Pol $\eta$ targeted siRNA also prevented TLS to a greater extent than our expectations. Forth, although siRNA to Pol $\iota$ had no detectable effect, that to Pol $\kappa$ delayed UV-TLS. To our knowledge, this is the first study reporting apparent evidence for the participation of Pol $\kappa$ in UV-TLS.

\section{Introduction}

Multiple systems have evolved to manage the genomic photoproducts generated by harmful UV light. One such system is nucleotide excision repair (NER), which eliminates photoproducts from DNA strands by dual incision on both sides of a damaged base. The NER system cannot, however, remove all UV-damaged bases. When a replicative DNA polymerase stalls upon encountering a residual photoproduct on the template strand, it is relieved by other low-processivity polymerase(s), which incorporate nucleotide(s) opposite the lesion, extend by a few nucleotides and dissociate from the $3^{\prime}-\mathrm{OH}$. The replicative polymerase then resumes DNA synthesis. This process, termed translesion replication (TLS) or replicative bypass (reviewed in [1]), is also one of the subtle systems that have evolved for the management of genomic photoproducts.

UV-C (100-290 nm wavelength) induces 2 main photoproducts [2]: the more frequent cyclobutane pyrimi- dine dimer (CPD) and the several-fold lower pyrimidinepyrimidone (6-4) photoproduct ((6-4)PP). cis-syn CPD, a predominant form of the multiple configurations, contains 2 adjacent pyrimidines that are covalently linked in parallel. Although the frequency of CPD varies with nucleotide composition, a ratio of $\mathrm{T}-\mathrm{T}$ to $\mathrm{C}-\mathrm{T}$ to $\mathrm{T}-\mathrm{C}$ to $\mathrm{C}-\mathrm{C}$ of $68: 13: 16: 3$ is obtained from UV-irradiated plasmid DNA. Cytosines within CPD are unstable, and are deaminated to uracil or 5methylcytosine, and further deaminated to thymine [3]. The helical distortion caused by CPD is so inconspicuous that almost half of the lesions remain unrepaired by NER, even 6 hours after UV irradiation in the case of CHO cells [2].

The (6-4)PPs from T-C, C-C, and, less frequently, T-T sequences are detected in UV-irradiated DNA whereas that of $\mathrm{C}-\mathrm{T}$ are not. In (6-4)PP, linkage between C- 6 of one pyrimidine and C- 4 of the adjacent pyrimidine cause the 2 bases to be in nearly perpendicular position. Consequently, formation of this lesion causes a major distortion in the double helix. NER preferentially removes (6-4)PP more 
rapidly than it removes CPD from the genome in human and rodent cells [4].

At least 5 mammalian DNA polymerases are suggested to be implicated in UV-induced TLS: Pols $\eta, \iota, \zeta, \kappa$, and Rev1, all of which belong to the $\mathrm{Y}$ family except for Pol $\zeta$ (B family) (reviewed in $[1,5,6])$. However, the participating polymerases and their roles have not been entirely characterized.

Patients with the autosomal recessive disorder, xeroderma pigmentosum variant $(\mathrm{XP}-\mathrm{V})$, have a predisposition to skin cancer, and XP-V cells demonstrate hypermutability after UV irradiation (reviewed in [7]). The defective gene in $\mathrm{XP}-\mathrm{V}$ encodes Pol $\eta$, which was first purified from a HeLa cell extract as an activity that complements TLS defect in XP-V cell extract [8]. Human Pol $\eta$ catalysed DNA synthesis past TT-CPD very efficiently and in a relatively accurate manner, as demonstrated by the lesion-bypass assay [7,9]. When template DNA contained a (6-4)TT-PP, Pol $\eta$ incorporated one (random) nucleotide opposite the first thymine and another nucleotide opposite the second thymine of the lesion, but rarely continued across the lesion [7, 9].

Human Pol $\eta$ was also identified via a search for the homolog of yeast Saccharomyces cerevisiae Rad30 gene, which encodes an error-free bypass protein [10]. Various XP-V causative mutations have been found in the Pol $\eta$ gene, hRAD30A, of XP-V patients [10, 11].

Pol $\iota(R A D 30 B)$ is the other mammalian homolog of yeast Pol $\eta$, isolated by a similar approach [12]. In contrast to $\mathrm{Pol} \eta$, Pol $\iota$ is less efficient and less accurate [13].

Pol $\kappa$ was obtained by cloning of a human homolog of the E. coli $\operatorname{din} B$ gene, encoding DNA Pol IV [14]. Pol $\kappa$ was reported to be unable to bypass either CPD or (6-4)PP $[15,16]$.

Originally, Rev1, 3, and 7 were cloned from S. cerevisiae isolates, in which the frequency of UV-induced reversion from $c y c 1$ mutations was reduced [17]. Human and mouse homologs (Rev1, 3, 7 gene) were later isolated [18-20]. Pol $\zeta$ is a comprex of the Rev3 and Rev7 gene products, which act as the catalytic and regulatory subunit, respectively. Yeast $\mathrm{Pol} \zeta$ is shown to be responsible for $98 \%$ of UV-induced base substitutions and $90 \%$ of frameshift mutations, in addition to spontaneous mutations [21]. Nonetheless, yeast Pol $\zeta$ itself was revealed to be too faithful to incorporate opposite CPD. Instead, it can efficiently extend from a matched or mismatched 3 '-end (reviewed in $[5,6]$ ). Human or mouse $\mathrm{Pol} \zeta$ is assumed to have similar enzymatic properties to that of yeast, because several lines of antisense RNA expression or siRNA knockdown experiments in human or mouse cells have proven that $\mathrm{Pol} \zeta$ is involved in mutagenic TLS [22-24].

Yeast and human Rev1 encode highly specialized DNA polymerases that preferentially insert a $\mathrm{C}$ residue opposite an abasic site in the template. This deoxycytidyl (dC) transferase activity is, however, unlikely to be required for UV-TLS, judging by observation in a yeast rev1-1 mutant strain [25], which retains much of its $\mathrm{dC}$ transferase activity, but has a missense mutation (G193R) in the N-terminal BRCT domain. Rev1 protein also contains ubiquitin-binding motifs (UBMs) that interact with monoubiquitinated PCNA (a DNA polymerase sliding clamp) [26]. In the downstream Cterminal region, Rev1 interacts not only with Rev7 but also with other bypass-polymerases [27], suggesting that Rev1 acts as a mediator and physical bridges between PCNA and Polל.

Following DNA damage, such as that caused by UV and MMS, monoubiquitins are conjugated to PCNA arrested at the lesion-site by RAD6/RAD18 and recruit bypasspolymerases $[28,29]$. In addition to Rev1, Pols $\eta, \iota$, and $\kappa$ possess UBMs and physically interact with PCNA $[1,5,6]$. Stalled replicative Pol $\delta$ is replaced, in turn, with one of these bypass polymerases bound on the PCNA by yet unknown "polymerase switching" mechanisms (reviewed in $[1,30]$ ).

Translesion replication is typically detected with an alkaline sucrose density gradient centrifugation (ASDG) technique. Pulse-labelled replication products are smaller in UVirradiated XP-V cells than in unirradiated cells; however, on prolonged incubation, the replication products in the irradiated cells eventually attain a high molecular weight similar to that in unirradiated cells. This conversion is interpreted that DNA synthesis is temporarily retarded by UV photoproducts, and then continues beyond the lesion, leaving a gap that is subsequently sealed [31]. The initial size of the newly synthesized DNA is approximately equal to the average distance between lesions in the template strands [32]. This means the gaps in the newly synthesized DNA are opposite the photoproducts [33]. Therefore, sealing of the gaps, by translesion or other postreplication repair mechanisms, can be observed by monitoring the molecular weight of labelled DNA.

Using a modified ASDG technique [34], we precisely detected the elongation of pulse-labelled replication products in the irradiated XP-V cells, showing that UV-TLS is delayed in the cells, but not completely abolished [35]. The marginal TLS is markedly prevented by caffeine at millimolar concentrations, as Lehmann et al. pointed out [31], and by proteasome inhibitors as well (unpublished results). In contrast, these agents do not retard UV-TLS in normal diploid cells. To know more about the inefficient polymerase(s) in vivo, we added specific DNA polymerase inhibitors. Butylphenyldeoxyguanosine (BuPGdR) inhibited TLS in XP-V cells [35], suggesting that $\mathrm{Pol} \zeta$ may be involved in this Pol $\eta$-independent bypass.

We recently reported that caffeine or proteasome inhibitors inhibit UV-TLS also in human cancer cells [36], and that, similar to XP-V cells, UV-TLS was much slower than in normal cells. These results suggested that UV bypass in cancer cells is predominantly of the Pol $\eta$-independent type. Therefore, we expected that $\mathrm{Pol} \zeta$ plays a major part in UV-TLS in cancer cells. Although Pol $\eta$ exists in normal quantity in these cells, it was supposed to be inactivated by some reasons. We explored these hypotheses here.

\section{Materials and Methods}

2.1. Design of siRNAs. All siRNAs duplexes were synthesized by JBioS (Japan) (Table 1). We designed the sequences for siRNA according to the JBioS guide (http://www.JBioS.co .jp/RNAiselect.htm). Selection of target sequence for each siRNA proceeds as follows: first, an AAG or AAC sequence is found at least 75 nucleotides (nt) downstream from the start codon; the AAG (or AAC) and the following 18 nt sequence 
TABLE 1: siRNAs used.

\begin{tabular}{|c|c|c|c|}
\hline siRNA & Sense strand ${ }^{(a),(b)}$ & Antisense strand $^{(\mathrm{a}),(\mathrm{b})}$ & $\begin{array}{l}\text { Prevention of UV-TLS } \\
\text { (result) }\end{array}$ \\
\hline siRev3-A & gcuuuacaugagauacaaaTT & uuuguaucucauguaaagcTT & significant \\
\hline siRev3-B & gacaguuuucagucaagauTT & aucuugacugaaaacugucTT & significant \\
\hline siRev3-C & gagguaugauccugauauuTT & aauaucaggaucauaccucTT & significant \\
\hline siRev3-D & guauugacuuaugucggauTT & auccgacauaagucaauacTT & significant \\
\hline $\begin{array}{l}\text { siRev3cont-A } \\
\text { (6nt mismatches) }\end{array}$ & gguuugaguaauguacgauTT & aucguacau uacucaaaccTT & no effect \\
\hline $\begin{array}{l}\text { siRev3cont-B } \\
\text { (4nt mismatches) }\end{array}$ & ggauugaguuauguacgauTT & aucguacauaacucaauccTT & no effect \\
\hline $\begin{array}{l}\text { siRev3cont-C } \\
\text { (2nt mismatches) }\end{array}$ & guauugaguuauguccgauTT & aucggacauaacucaauacTT & no effect \\
\hline siRev7-A & gauccaggucaucaaggauTT & auccuugaugaccuggaucTT & partial \\
\hline siRev7-B & gaugcagcuuuacguggaaTT & uuccacguaaagcugcaucTT & significant \\
\hline siRev7-C & cacugucugucucaaauacTT & guauuugagacagacagugTT & significant \\
\hline siRev7cont-A & gaugcagguuuacgucgaaTT & uucgacguaaaccugcaucTT & no effect \\
\hline siRev1-A & cacauauuauugccacaaaTT & uuuguggcaauaauaugugTT & significant \\
\hline siRev1-B & gaagauugaaacggaaaauTT & auuuuccguuucaaucuucTT & significant \\
\hline siRev1-C & ccuucagacugcaauuuuaTT & uaaaauugcagucugaaggTT & significant \\
\hline siRev1-D & gugugaauugacugaguuuTT & aaacucagucaauucacacTT & significant \\
\hline siRevlcont-E & ccuucacaccgcaacguuaTT & uaacguugcggugugaaggTT & little to no effect \\
\hline siPol $\eta-4$ & uaaaccuugugcaguuguaTT & uacaacugcacaagguuuaTT & partial \\
\hline siPol $\eta-A$ & gaaguuauguccagaucuuTT & aagaucuggacauaacuucTT & significant \\
\hline siPol $\eta-B$ & gcuucgcuuucaucucuuaTT & uaagagaugaaagcgaagcTT & significant \\
\hline siPol $\eta$ cont-A & uaaaccuggugcagucguaTT & uacgacugcaccagguuuaTT & no effect \\
\hline siPol $t-\mathrm{A}$ & gccucauacagugagauuaTT & uaaucucacuguaugaggcTT & no effect \\
\hline siPol $t-5$ & aaguguccacaguugguauTT & auaccaacuguggacacuuTT & no effect \\
\hline siPol $\kappa-A$ & gagaaaauuaacaaaauuaTT & uaauuuuguuaauuuucucTT & partial \\
\hline siPol $\kappa-B$ & gaauaaaccaaauggacaaTT & uuguccauuugguuuauucTT & partial \\
\hline siPol $\kappa-C$ & cuguuaccauuaaguugaaTT & uucaacuuaaugguaacagTT & considerable \\
\hline siPol $\kappa$ cont-B & gagaaaaguaacaagcuuaTT & uaagcuuguuacuuuucucTT & little to no effect \\
\hline
\end{tabular}

(a) Small letters mean ribonucleotides, and large letters mean deoxyribonucleotides.

(b) Mismatched bases are illustrated in bold and italic letters.

(N18) are picked up. Ideally, the $2 \mathrm{nt}$ following the N18 are TT, TN, NT, or AA. GC content of N18 is recommended to be about $40 \%$ to $50 \%$. If GC content is less than $30 \%$, or greater than $70 \%$, avoid the sequence. The N18 had better be AT-rich in the $3^{\prime}$ half and especially at the $3^{\prime}$ end (2 nt).

2.2. HeLa Cell Culture and siRNA Treatment. HeLa cells were maintained in monolayers in Dulbecco's modified Eagle's medium (D'MEM) supplemented with $10 \%$ fetal calf serum (FCS) ("normal" medium), trypsinized and seeded into culture dishes $\left(2 \times 10^{5}\right.$ cells $/ \phi 60 \mathrm{~mm}$ dish $)$. About $6-8$ hours later, the cells were treated with micelles of siRNA and OligofectAMINE (Invitrogen), formed according to the manufacturer's protocol, except Opti-MEM was replaced with D'MEM. The siRNA concentration used in RT-PCR analysis and western blot analysis was $5 \mathrm{nM}$.

2.3. UV Irradiation and Translesion Replication. Forty hours after siRNA addition, HeLa cells were exposed to UV light $\left(10 \mathrm{~J} / \mathrm{m}^{2}\right)$ from a germicidal lamp (Toshiba GL15) at
$0.6 \mathrm{~J} / \mathrm{m}^{2}$ per s. After 30 minutes in culture, the medium was changed to labeling medium consisting of D'MEM supplemented with $10 \% \mathrm{FCS}$ and $10 \mu \mathrm{Ci} / \mathrm{mL}$ of $\left[U-{ }^{14} \mathrm{C}\right]$ thymidine (Moravek MC267, $470 \mathrm{mCi} / \mathrm{mmol}$ ). UV-irradiated cells were pulse-labelled for 1 hour, while nonirradiated cells were labelled for 30 minutes. The medium was changed to normal medium, and the cells were chased for 5 hours. These cells were harvested by trypsinisation and examined by ASDG [34].

2.4. Alkaline Sucrose Density Gradient Centrifugation (ASDG). Cells (about $1 \times 10^{5}$ in $50 \mu \mathrm{L}$ PBS) were gently layered onto $50 \mu \mathrm{L}$ of $1 \%$ sucrose in PBS, which was overlaid on $100 \mu \mathrm{L}$ of lysis solution $(0.6 \mathrm{M} \mathrm{KOH}, 2.0 \mathrm{M} \mathrm{KCl}, 10 \mathrm{mM}$ EDTA, and $1 \% N$-lauroylsarcosine), which was placed on top of a $4.35 \mathrm{~mL}$ alkaline $5-20 \%$ sucrose gradient $(0.3 \mathrm{M} \mathrm{KOH}$, $2.0 \mathrm{M} \mathrm{KCl}, 1 \mathrm{mM}$ EDTA, and $0.1 \% \mathrm{~N}$-lauroylsarcosine) with $0.4 \mathrm{~mL}$ of alkaline $80 \%$ sucrose as a cushion at the bottom. The gradient was centrifuged at $6,000 \mathrm{rpm}(4,320 \times \mathrm{g})$ for 15.6 hours at $15^{\circ} \mathrm{C}$ in a Beckman SW 50.1 rotor. The gradient was fractionated onto 30 circles of no. 17 paper 
(Whatman). The paper circles were dried, immersed in cold $5 \%$ trichloroacetic acid for 10 minutes, washed 3 times with ethanol and once with acetone, and dried; radioactivity was then measured. As a molecular weight marker, $\left[{ }^{14} \mathrm{C}\right]$-labelled T4 DNA phage particles were placed on the lysis layer and sedimented in a parallel run. The approximate fragment length of each fraction was estimated on the basis of the position of the T4 DNA marker and that of E. coli DNA and adjusted by sucrose density curve [34]. Average fragment length (in megabase $(\mathrm{Mb})$ ) of each profile is shown in Figures as fragment length of the median fraction [35]. (Median fraction is the middle fraction that separates the higher half of the profile from the lower half.)

2.5. RT-PCR. Total RNA was isolated using an RNeasy spin column (QIAGEN). One $\mu \mathrm{g}$ of total RNA was treated with DNase I (Invitrogen), reverse-transcribed using SuperScript II (Invitrogen) with random hexamers or PrimeScript II (TaKaRa) with oligo(dT) primers, followed by treatment with E. coli RNase $\mathrm{H}$ (Invitrogen). The PCR mixture contained cDNA templates, dNTPs, rTaq Pol (TOYOBO), anti-Taq (TOYOBO), and appropriate primers. Primers to assess knockdown of each gene were as follows: Rev3, 5'-GGAACGTCAACAGGAGCAAC-3' and 5'-GGAGCAAATCCAACACCTGC-3'; Rev7, 5'-TGCTGTCCATCAGCTCAGAC- $3^{\prime}$ and $5^{\prime}$-AGAGCACTTGGAATCAGGGC$3^{\prime}$; Rev1, 5'-CTCCTGCAGAGAAACCCCTG-3' and $5^{\prime}-$ ACAAGCACTTATGGCACAGCT-3'; Pol $\eta$, 5'-CCCAGGCAACTACCCAAAAC- $3^{\prime}$ and $5^{\prime}$-GGGCTCAGTTCCTGTACTTTG-3'; Pol $\iota$, 5'-ATGATCAAGTGTTGCCCACAC$3^{\prime}$ and $5^{\prime}$-ACATGACCCGACACAGTCAC- $3^{\prime}$; Pol $\kappa, 5^{\prime}-$ AGACAAGAATACCGCCAGCC- $3^{\prime}$ and $5^{\prime}$-AGGAAGGATTATTGCACTTGCC-3'; GAPDH, 5' -ACCACAGTCCATGCCATCAC- $3^{\prime}$ and $5^{\prime}$-TCCACCACCCTGTTGCTGTA- $3^{\prime}$. All PCR reactions were carried out for 27 cycles, with the exception of GAPDH ( 25 cycles). PCR products were subjected to a MultiNA microtip electrophoresis DNA/RNA analyzer (Shimadzu Biotech).

2.6. Western Blotting. Cells were rinsed with PBS, lysed with $1 \times$ SDS-PAGE loading buffer and collected by scraping. Cell lysate was boiled for 10 minutes, sonicated for 30 seconds and centrifuged at $20,000 \times \mathrm{g}$ for 10 minutes. The supernatant was used for western blotting after measuring protein content by Bradford method. The supernatant ( $60 \mu \mathrm{g}$ protein) was separated on 5-20\% (Rev7, Rev1 and Pol $\iota$ ) or $3-10 \%$ (the rest) SDS-polyacrylamide gels (Wako) and transferred onto Immobilon- $\mathrm{P}^{\mathrm{SQ}}$ membranes (Millipore). The membranes were incubated with antibodies to bypasspolymerases (Rev1, Santa Cruz Biotechnology sc-48806; Rev7, BD Transduction Laboratories 612266; Pol $\eta$, Santa Cruz Biotechnology sc-17770; Pol $\iota$, Abnova H00011201M01; Pol $\kappa$, a kind gift from Dr. Tomoo Ogi, Nagasaki University), and then with horseradish peroxidase-conjugated secondary antibodies (anti mouse IgG, DAKO PO-0447; antirabit IgG, DAKO PO-0448). Antibodies were diluted with CanGetSignal (TOYOBO) to enhance immunoreactivity. The signals were developed with ECL-Plus (GE Healthcare), and blots were stripped for reprobing with anti $\alpha$-tubulin antibody (Sigma T5168).

\section{Results}

3.1. Effect of siRNAs to Rev3 or Rev7 on HeLa UV-TLS. We selected 4 target sites from the human Rev 3 mRNA sequence (NCBI locus: NM_002912), according to the JBioS guide. All the Rev3 siRNAs (Table 1) effectively knocked down expression of Rev3 (Figure 1(a)) and, at $5 \mathrm{nM}$, abolished UV-TLS in HeLa cells (Figure 1(b)). Replication products immediately after UV irradiation were sedimented as a sharp peak, illustrated by a thin line in Figure 1(b), slightly larger in size than the T4 phage DNA marker. When only Oligofectamine-treated cells were chased in normal medium for 5 hours, the products joined to form larger DNA with lengths in the order of megabases $(\mathrm{Mb})$, illustrated by a thick line. In Rev3 siRNA-transfected cells, the products remain in smaller size, as depicted by a thin line with open marks, demonstrating these siRNAs prevent UV-TLS (Figure 1(b)).

We assessed how many mismatched nucleotides (nt) are necessary at minimum for the negative control siRNA (Figure 1(c)) and found that siRev3cont-C, designed from siRev3-D with $2 \mathrm{nt}$ mismatches, did not prevent UV-TLS, indicating that these Rev3 siRNAs degrade Rev3 mRNA with high specificity. The dose-response profile of siRev3-D, shows that only $1 \mathrm{nM}$ siRNA sufficiently inhibited UV-TLS (Figure 1(d)). The siRev3-D siRNA had no effect on normal replication (Figure $1(\mathrm{e})$ ).

We selected 3 target sites from the human Rev7 mRNA sequence (NM_006341) (Table 1), and found that all siRNAs effectively reduced $\operatorname{Rev} 7$ expression (Figure 2(a)); $5 \mathrm{nM}$ siRev7-B or siRev7-C completely abolished UV-TLS (siRev7-A elicited only partial prevention) (Figure 2(b)). The siRev7cont-A, designed from siRev7-B with $2 \mathrm{nt}$ mismatches, had no effect (ibid). The dose-response profile of siRev7$B$ shows that $1 \mathrm{nM}$ siRNA was sufficient to inhibit UVTLS (Figure 2(c)); Rev7 siRNAs had no effect on normal replication (Figure $2(\mathrm{~d})$ ).

3.2. SiRNAs to Rev1 Significantly Abrogated UV-TLS. For knockdown of Rev1 expression, we selected 4 sites from the human Rev1 mRNA sequence (NM_016316) (Table 1) (Figure 3(a)). The siRNAs ( $5 \mathrm{nM}$ ) targeted to these sites abolished UV-TLS in HeLa cells (Figure 3(b)). The siRev1cont$\mathrm{E}$, designed from siRev1-C with $4 \mathrm{nt}$ mismatches, had little to no effect. The dose-response profile of siRev1-C shows that $1 \mathrm{nM}$ of the siRNA was enough to inhibit UV-TLS (Figure 3(c)); siRev1-C or siRev1-D siRNA had no effect on normal replication (Figure 3(d)).

3.3. SiRNAs to Poln Prevented UV-TLS to a Great Extent. First, we tested the siRNA described by Choi and Pfeifer [37], siPol $\eta-4$, which partially inhibited UV-TLS (Figure 4(a)). The negative control, siPol $\eta$ cont-A, was designed from siPol $\eta$ - 4 with 2 nt mismatches, had no effect (ibid). We added 2 Pol $\eta$ siRNAs of new design (human Pol $\eta$ mRNA sequence: NM_006502) (Table 1), and these siRNAs also effectively 


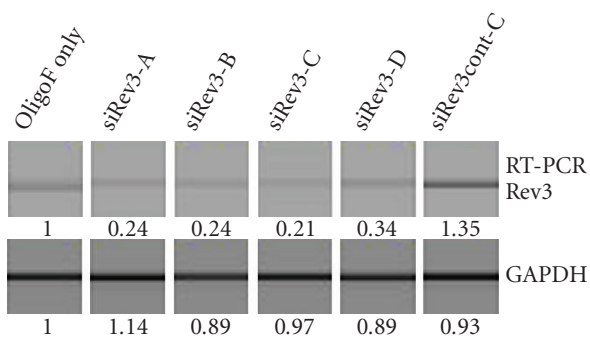

(a) RT-PCR
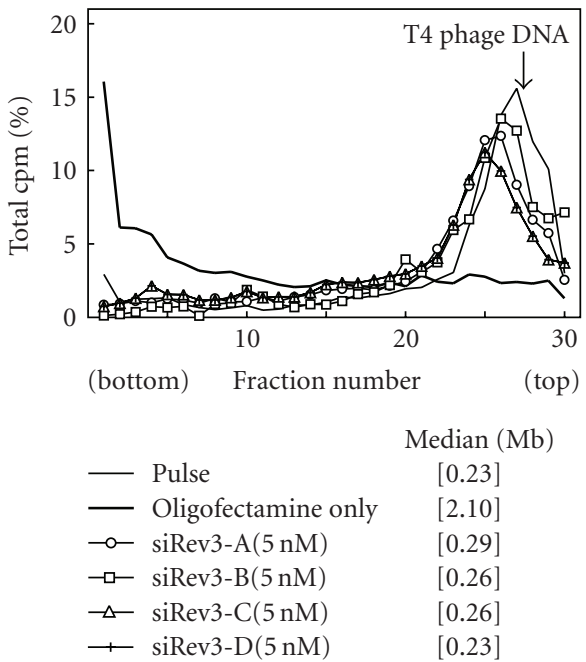

(b) Effects of various siRNAs (UV)

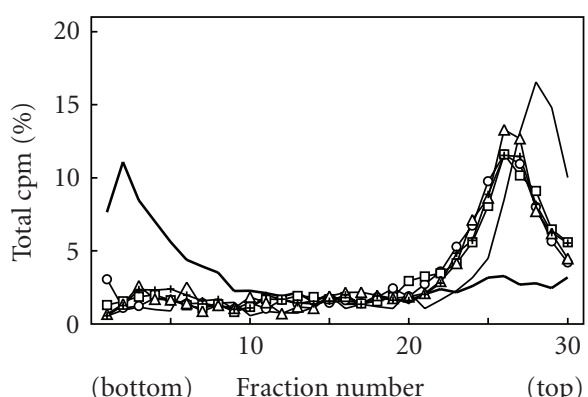

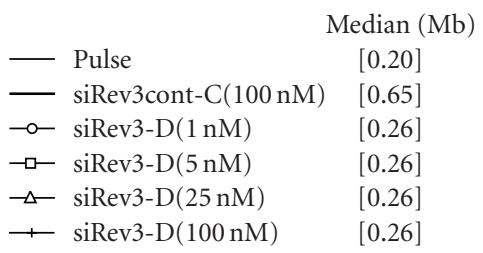

(d) Dose-response (siRev3-D)
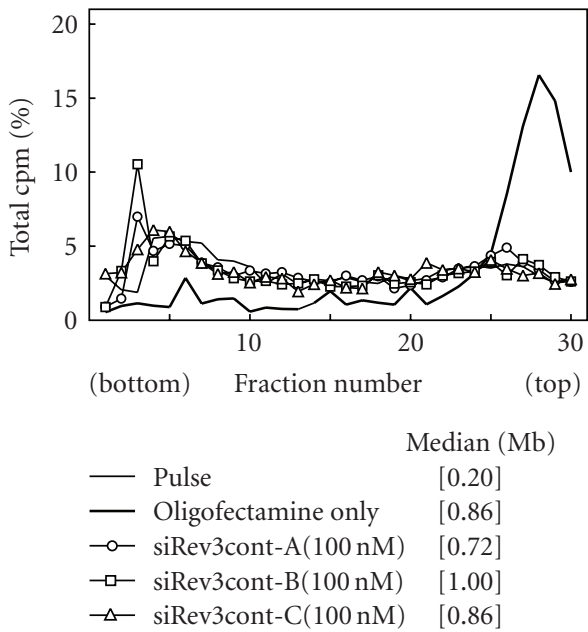

(c) Effects of control siRNAs (UV)
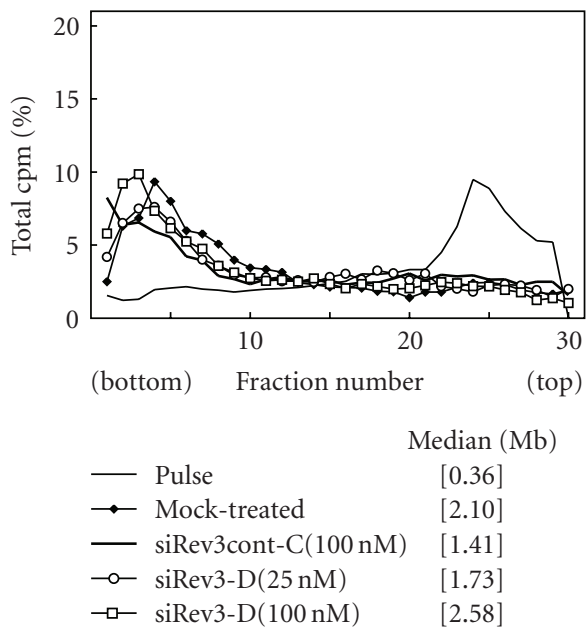

(e) Effect of various siRNAs (noUV)

FIGURE 1: Efficient knockdown by Rev3 siRNAs and their effects on UV-induced TLS in HeLa cells (ASDG profiles of replication products). (a) Efficiency of knockdown on Rev3 expression (RT-PCR analysis); (b) Effects of various Rev3 siRNAs; (c) Effects of Rev3 control siRNAs; (d) Dose-response of siRev3-D; (e) Effect of various Rev3 siRNAs (no UV control). Twenty-four hours after Rev3 siRNA transfection, total RNA was isolated and Rev3 RNA was quantified by RT-PCR. Results were shown in MultiNA gel images and the expression level was presented under the panel (a). Forty hours after Rev3 siRNA transfection, cells were UV-irradiated $\left(10 \mathrm{~J} / \mathrm{m}^{2}\right)$, incubated in normal medium for 30 minutes, pulse-labelled with $10 \mu \mathrm{Ci} / \mathrm{mL}$ of $\left[{ }^{14} \mathrm{C}\right]$ thymidine for 1 hour, washed twice with $\mathrm{PBS}$, and incubated for 5 hours at $37^{\circ} \mathrm{C}$ in normal medium (b, c, d). siRev3cont-A, 6 nt mismatches; siRev3cont-B, 4 nt mismatches; siRev3cont-C, 2 nt mismatches (c). Forty hours after Rev3 siRNA transfection, cells were not UV-irradiated, pulse-labelled with $10 \mu \mathrm{Ci} / \mathrm{mL}$ of $\left[{ }^{14} \mathrm{C}\right]$ thymidine for 30 minutes, washed twice with PBS, and incubated at $37^{\circ} \mathrm{C}$ in normal medium for 1 hour (e). Some of these profiles overlap (c, d, e). Sedimentation is from right to left. The arrow indicates the position of T4 phage DNA (166 kb, i.e., approximately $5.5 \times 10^{7} \mathrm{Da} /$ single strand). Labelled E.coli DNA (approximately $4 \mathrm{Mb}$ ) sedimented near the bottom (fractions 3-6) (4). Average fragment length (in Mb) of each profile is shown in square brackets as fragment length of the median fraction. 


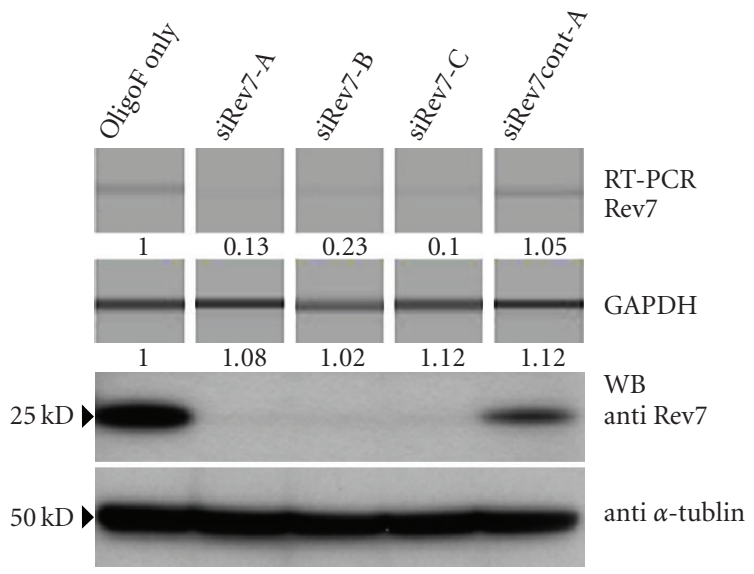

(a) RT-PCR and western blotting

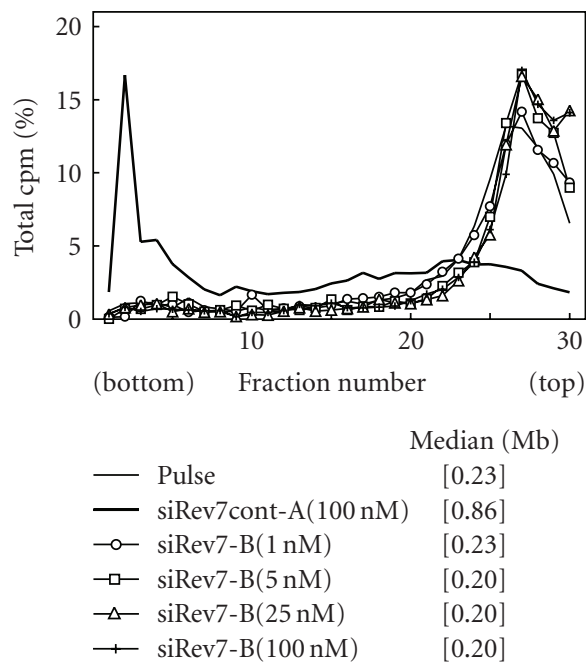

(c) Dose-response (siRev7-B)

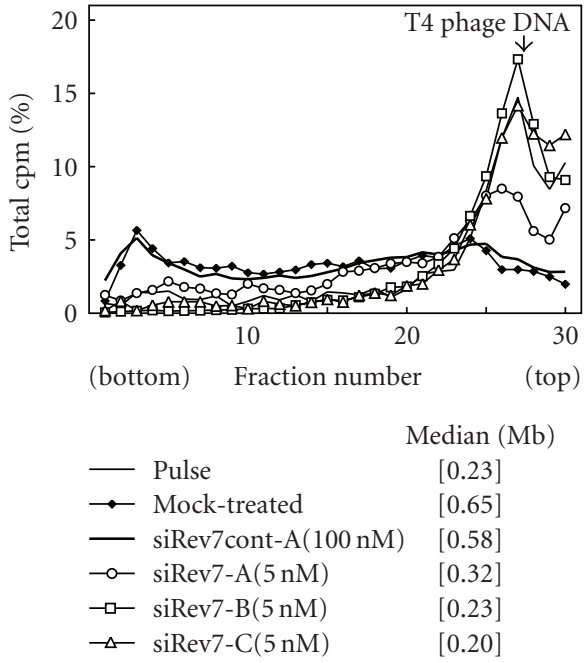

(b) Effects of various siRNAs (UV)

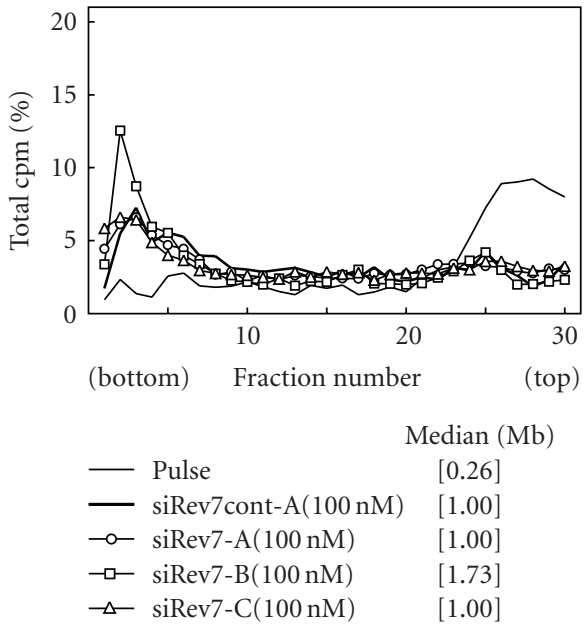

(d) Effect of various siRNAs (noUV)

FIGURE 2: Efficient knockdown by Rev7 siRNAs and their effects on UV-induced TLS in HeLa cells (ASDG profiles of replication products). (a) Efficiency of knockdown on Rev7 expression (RT-PCR analysis and western blot analysis); (b) Effects of various Rev7 siRNAs; (c) Doseresponse of siRev7-B; (d) Effect of various Rev7 siRNAs (no UV control). Twenty-four hours after Rev7 siRNA transfection, total RNA was isolated and Rev7 RNA was quantified by RT-PCR. Results were shown in MultiNA gel images and the expression level was presented under the panel (a). Forty hours after Rev7 siRNA transfection, whole cell extracts were prepared and Rev7 protein was quantified by western blot analysis (a). Forty hours after Rev7 siRNA transfection, cells were UV-irradiated $\left(10 \mathrm{~J} / \mathrm{m}^{2}\right)$, incubated in normal medium for 30 minutes, pulse-labelled with $10 \mu \mathrm{Ci} / \mathrm{mL}$ of $\left[{ }^{14} \mathrm{C}\right]$ thymidine for 1 hour, washed twice with PBS, and incubated for 5 hours at $37^{\circ} \mathrm{C}$ in normal medium $(\mathrm{b}$, c). Forty hours after Rev7 siRNA transfection, cells were not UV-irradiated, pulse-labelled with $10 \mu \mathrm{Ci} / \mathrm{mL}$ of $\left[{ }^{14} \mathrm{C}\right]$ thymidine for $30 \mathrm{minutes}$, washed twice with PBS, and incubated at $37^{\circ} \mathrm{C}$ in normal medium for 1 hour (d). Some of these profiles overlap (c, d). Sedimentation is from right to left. The arrow indicates the position of T4 phage DNA (166 kb, i.e., approximately $5.5 \times 10^{7} \mathrm{Da} /$ single strand). Average fragment length (in $\mathrm{Mb}$ ) of each profile is shown in square brackets.

knocked down Pol $\eta$ expression (Figure 4(b)). These latter siRNAs at $5 \mathrm{nM}$ abolished UV-TLS to a greater extent than siPol $\eta$-4 (Figure 4(c)). The Pol $\eta$ siRNAs had no effect on normal replication (Figure $4(\mathrm{~d})$ ).
3.4. Polk siRNAs Delayed UV-TLS, While siRNAs to Polı Did Not. Although Pol $\iota$ siRNAs efficiently prevented Pol $\iota$ expression (Figure 5(a)), we could not detect these effects on ASDG profiles (Figure 5(b)). The siPolı-5 was reported by 


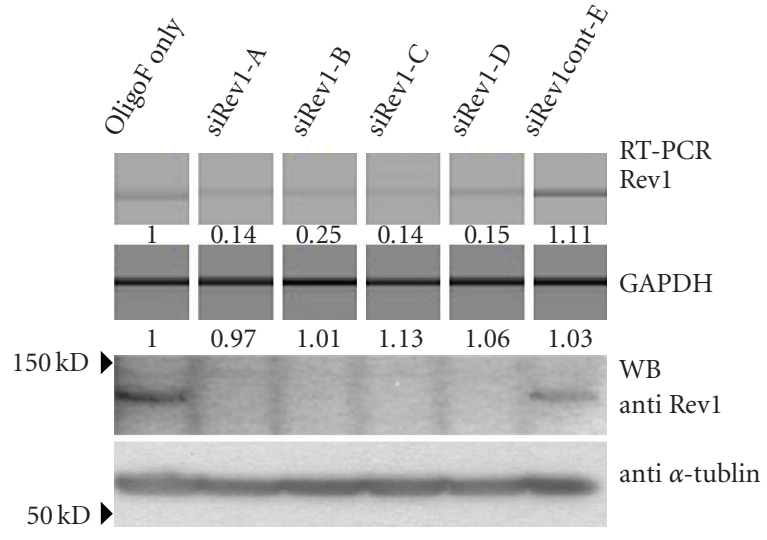

(a) RT-PCR and western blotting

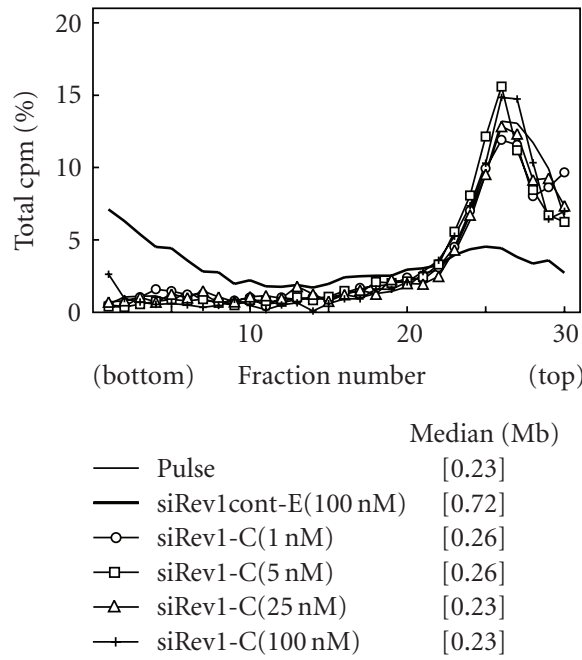

(c) Dose-response (siRev1-C)

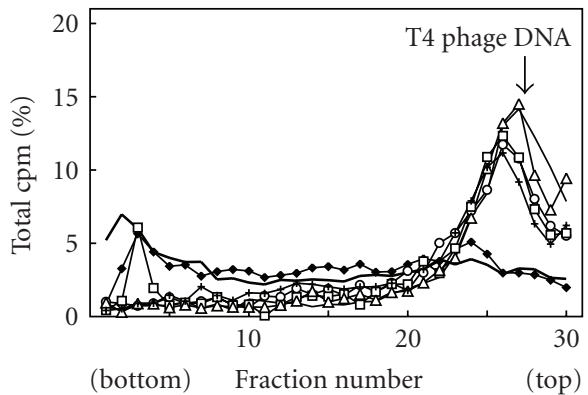

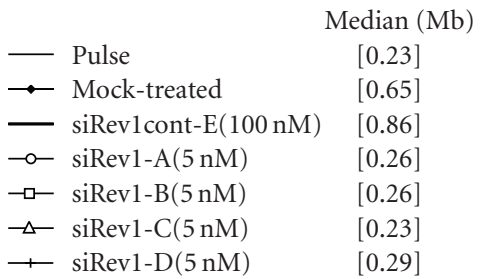

(b) Effects of various siRNAs (UV)

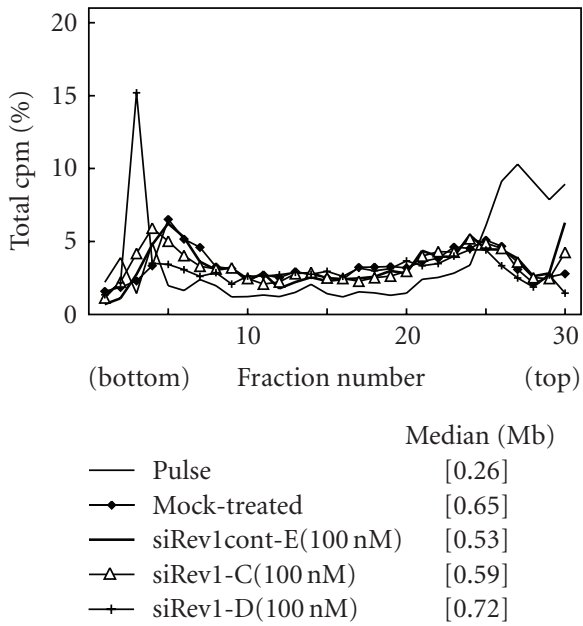

(d) Effect of various siRNAs (noUV)

Figure 3: Efficient knockdown by Rev1 siRNAs and their effects on UV-induced TLS in HeLa cells (ASDG profiles of replication products). (a) Efficiency of knockdown on Rev1 expression (RT-PCR analysis and western blot analysis); (b) Effects of various Rev1 siRNAs on UV-TLS; (c) Dose-response of siRev1-C on UV-TLS; (d) Effect of various siRNAs (no UV control). Twenty-four hours after Rev1 siRNA transfection, total RNA was isolated and Rev1 RNA was quantified by RT-PCR. Results were shown in MultiNA gel images and the expression level was presented under the panel (a). Forty hours after Rev1 siRNA transfection, whole cell extracts were prepared and Rev1 protein was quantified by western blot analysis (a). Forty hours after Rev1 siRNA transfection, cells were UV-irradiated $\left(10 \mathrm{~J} / \mathrm{m}^{2}\right)$, incubated in normal medium for 30 minutes, pulse-labelled with $10 \mu \mathrm{Ci} / \mathrm{mL}$ of $\left[{ }^{14} \mathrm{C}\right]$ thymidine for 1 hour, then washed twice with $\mathrm{PBS}$, and incubated for 5 hours at $37^{\circ} \mathrm{C}$ in normal medium (b, c). Forty hours after Rev1 siRNA transfection, cells were not UV-irradiated, pulse-labelled with $10 \mu \mathrm{Ci} / \mathrm{mL}$ of $\left[{ }^{14} \mathrm{C}\right]$ thymidine for 30 minutes, washed twice with PBS, and incubated at $37^{\circ} \mathrm{C}$ in normal medium for 1 hour $(\mathrm{d})$. Some of these profiles overlap (b, c). Sedimentation is from right to left. The arrow indicates the position of T4 phage DNA (166 kb, i.e., approximately $5.5 \times 10^{7} \mathrm{Da}$ /single strand). Average fragment length (in $\mathrm{Mb}$ ) of each profile is shown in square brackets.

Choi et al. [38], and the target sequence of siPoll-A was $4 \mathrm{nt}$ downstream from the one reported by Machida et al. [39] (human Polı mRNA sequence: NM_007195).

We prepared 3 siRNAs for knockdown of Pol $\kappa$ mRNA (NM_016218) (Figure 6(a)). In contrast to siPoll, these Pol $\kappa$ siRNAs $(5 \mathrm{nM})$ delayed UV-TLS in HeLa cells
(Figure 6(b)). The siPol $\kappa$ cont-B, from siPol $\kappa-\mathrm{A}$ with $3 \mathrm{nt}$ mismatches, had little to no effect. The doseresponse profile of siPol $\kappa$-A shows that the molecule sufficiently inhibited UV-TLS at $1 \mathrm{nM}$ (Figure 6(c)). The Pol $\kappa$ siRNAs had no effect on normal replication (Figure 6(d)). 


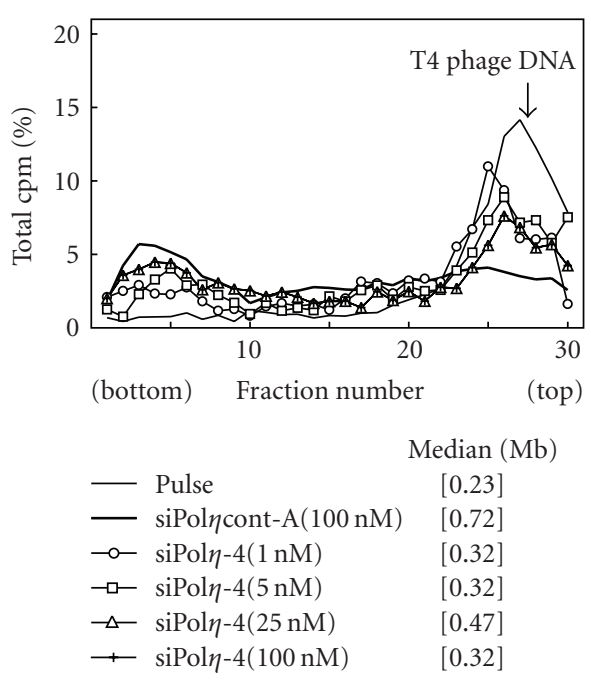

(a) Dose-response (siPol $\eta-4)$

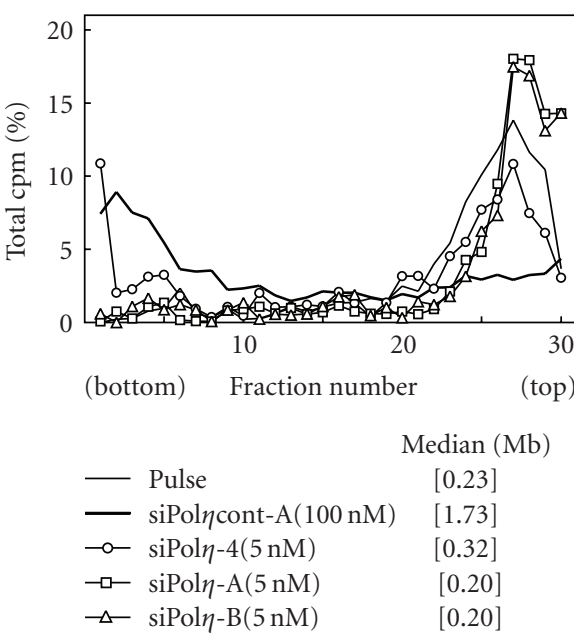

(c) Effects of various siRNAs (UV)

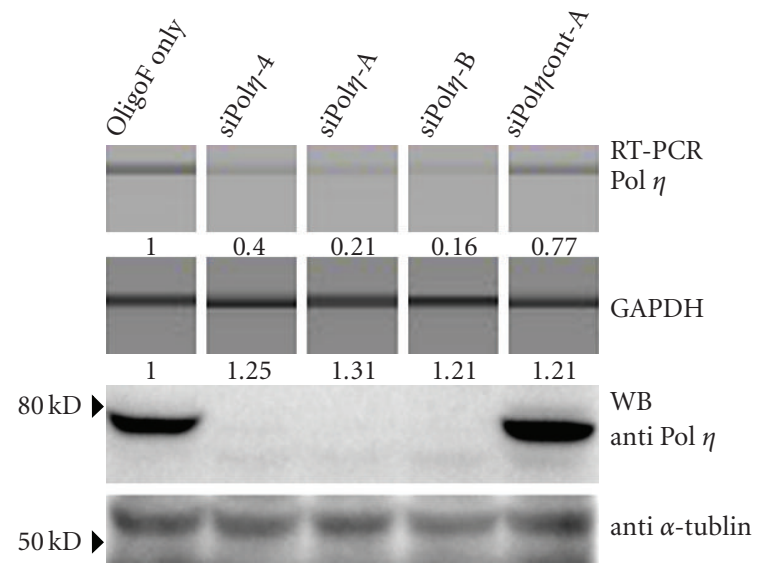

(b) RT-PCR and western blotting

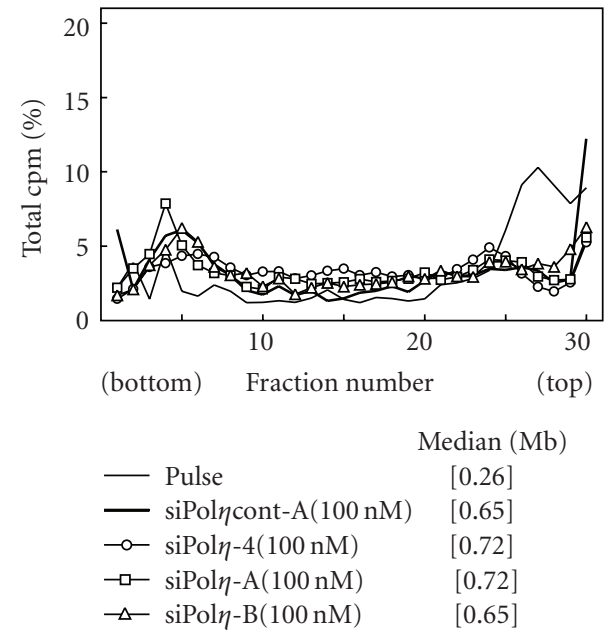

(d) Effect of various siRNAs (noUV)

FIGURE 4: Efficient knockdown by Pol $\eta$ siRNAs and the effects on UV-induced TLS in HeLa cells (ASDG profiles of replication products). (a) Dose-response of siPol $\eta$-4 on UV-TLS, Efficiency of knockdown on Pol $\eta$ expression; (b) RT-PCR analysis and western blot analysis, (c) Effects of various Pol $\eta$ siRNAs on UV-TLS, (d) Effect of various Pol $\eta$ siRNAs (no UV control). Twenty-four hours after Pol $\eta$ siRNA transfection, total RNA was isolated and Pol $\eta$ RNA was quantified by RT-PCR. Results were shown in MultiNA gel images and the expression level was presented under the panel (b). Forty hours after Pol $\eta$ siRNA transfection, whole cell extracts were prepared and Pol $\eta$ protein was quantified by western blot analysis (b). Forty hours after Pol $\eta$ siRNA transfection, cells were UV-irradiated $\left(10 \mathrm{~J} / \mathrm{m}^{2}\right)$, incubated in normal medium for 30 minutes, pulse-labelled with $10 \mu \mathrm{Ci} / \mathrm{mL}$ of $\left[{ }^{14} \mathrm{C}\right]$ thymidine for 1 hour, then washed twice with PBS, and incubated for 5 hours at $37^{\circ} \mathrm{C}$ in normal medium $(\mathrm{a}, \mathrm{c})$. Forty hours after Pol $\eta$ siRNA transfection, cells were not UV-irradiated, pulse-labelled with $10 \mu \mathrm{Ci} / \mathrm{mL}$ of $\left[{ }^{14} \mathrm{C}\right]$ thymidine for 30 minutes, washed twice with $\mathrm{PBS}$, and incubated at $37^{\circ} \mathrm{C}$ in normal medium for 1 hour $(\mathrm{d})$. Some of these profiles overlap (d). Sedimentation is from right to left. The arrow indicates the position of T4 phage DNA (166 kb, i.e., approximately $5.5 \times 10^{7} \mathrm{Da}$ /single strand). Average fragment length (in $\mathrm{Mb}$ ) of each profile is shown in square brackets.

\section{Discussion}

We verified the involvement of multiple bypass polymerases in UV-TLS in HeLa cells using original siRNAs and ASDG technique, which is consistent with the recent model of 2 polymerase mechanisms [40, 41]. Rev3 and Rev7, which comprise Pol $\zeta$, were confirmed to participate in mutagenic UV-TLS. Also, Rev1 was suggested to play an important role 


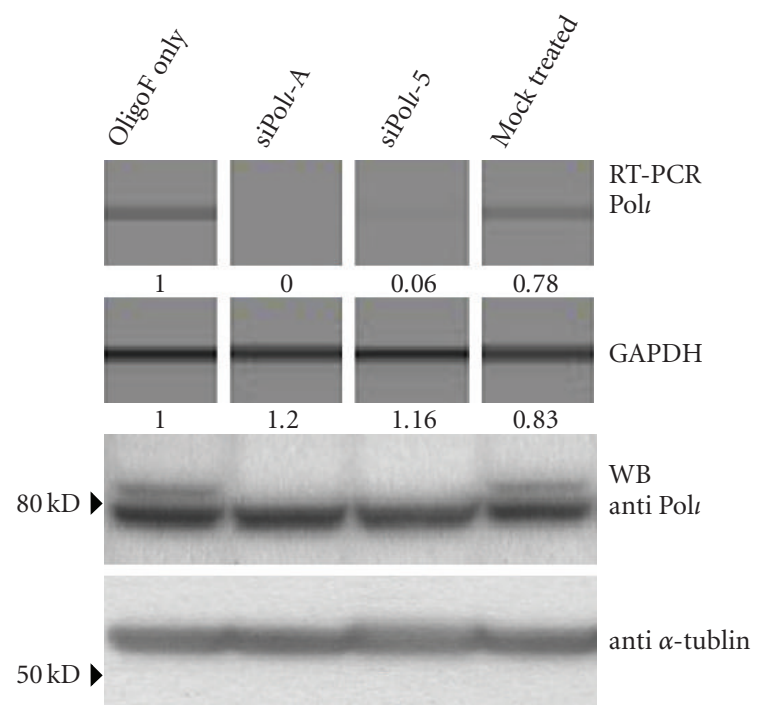

(a) RT-PCR and western blotting

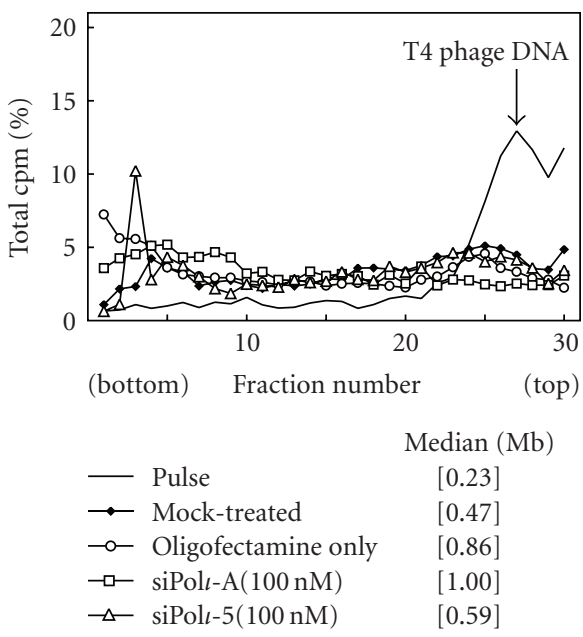

(b) Effects of various siRNAs (UV)

FIGURE 5: Efficient knockdown by Pol $\iota$ siRNAs and the effects on UV-induced TLS in HeLa cells (ASDG profiles of replication products). (a) Efficiency of knockdown on Polı expression (RT-PCR analysis and western blot analysis); (b) Effects of two Pol $\iota$ siRNAs on UV-TLS. Twentyfour hours after Pol $\iota$ siRNA transfection, total RNA was isolated and Pol $\iota$ RNA was quantified by RT-PCR. Results were shown in MultiNA gel images and the expression level was presented under the panel (a). Forty hours after Pol $\iota$ siRNA transfection, whole cell extracts were prepared and Pol $\iota$ protein was quantified by western blot analysis (a). Forty hours after Pol $\iota$ siRNA transfection, cells were UV-irradiated $\left(10 \mathrm{~J} / \mathrm{m}^{2}\right)$, incubated in normal medium for 30 minutes, pulse-labelled with $10 \mu \mathrm{Ci} / \mathrm{mL}$ of $\left[{ }^{14} \mathrm{C}\right]$ thymidine for 1 hour, then washed twice with PBS, and incubated for 5 hours at $37^{\circ} \mathrm{C}$ in normal medium (b). Sedimentation is from right to left. The arrow indicates the position of T4 phage DNA (166 kb, i.e., approximately $5.5 \times 10^{7} \mathrm{Da} /$ single strand). Average fragment length (in Mb) of each profile is shown in square brackets.

in human TLS, although in avian DT40 cells, Rev1 may have a distinct role [42]. We were surprised to find that siRNAs against Pol $\eta$ prevented TLS to a great extent. TLS was delayed in Pol $\kappa$ siRNA-transfected cells, but not in siPol-transfected cells.

We anticipated a limited participation of Pol $\eta$, because UV-TLS in HeLa cells is very slow (i.e., inefficient) and caffeine-sensitive [35]. However, siRNAs against Pol $\eta$, particularly siPol $\eta$-A and siPol $\eta$-B, prevented TLS to a great extent. Since both Rev3 and Rev7 siRNAs also significantly abolished UV-TLS, these results suggest that the Pol $\zeta$-dependent TLS pathway and the Pol $\eta$-dependent process are not mutually exclusive but overlapped.

Enzymology of yeast $\mathrm{Pol} \zeta$ revealed that this polymerase is too faithful to insert nucleotides opposite a CPD, although it efficiently extends from a matched or mismatched $3^{\prime}$ end $[5,6]$. Therefore, we assumed that mutagenic (errorprone) TLS proceeded through the insertion by Pol $\iota$ or Pol $\kappa$ of mismatched nucleotides opposite UV photoproducts, followed by extension by Pol $\zeta$. Our data showed, however, that siPolı had no effect, and siPol $\kappa$ partially prevented TLS. These results suggest that in some cases, Pol $\eta$, and to a lesser extent Polk, may insert nucleotide(s) opposite UV photoproducts, followed by extension by Pol $\zeta$.

Pol $\eta$ is capable of bypassing a CPD without aid of other TLS polymerases. Both yeast and human Pol $\eta$, however, incorporate wrong nucleotide at a fairly high rate and can extend these mismatched primer termini with only a frequency of $\sim 10^{-2}$ to $10^{-3}$ relative to extension from matched primer termini $[6,43]$. Plausibly, Pol $\eta$ dissociates from there and the proof-reading exonuclease of $\operatorname{Pol} \delta$ removes the wrong nucleotide [44]. To the primer termini, Pol $\eta$ is recruited again and incorporates a new nucleotide. This cycle is repeated until Pol $\eta$ incorporates a correct nucleotide. We suppose that disruption or malfunction of this cooperation renders mismatched primer termini accessible to Pol $\zeta$.

Recently, Yoon et al. published 2 papers describing the effects of siRNA knockdown on the efficiency of TLS at TTCPD [45] or (6-4)TT PP [46] on duplex plasmids in human cells. They also reported the effects of siRNA knockdown on mutation frequencies in the $\lambda$ phage $c I I$ gene lysogenized in mouse cells expressing a (6-4)PP photolyase [45] or CPD photolyase [46]. The results of this tremendous and detailed study demonstrated that Pols $\eta, \kappa$, and $\zeta$ contribute to CPD bypass, wherein Pols $\kappa$ and $\zeta$ promote mutagenic TLS and Pol $\eta$ executes error-free bypasses (Pol $\iota$ siRNA had no effect) [45]. As for (6-4)PP bypass, Pols $\eta$ and $\iota$ provide alternate pathways for mutagenic TLS, and Pol $\zeta$ acts in a predominantly error-free manner (Pol $\kappa$ siRNA had no effect) [46].

The participation of Pols $\kappa$ or $\iota$ in CPD bypass was similarly demonstrated by our results and those of Yoon et al. [45]. Because (6-4)PP is a minor photoproduct, which is removed predominantly by NER, and because HeLa cells 


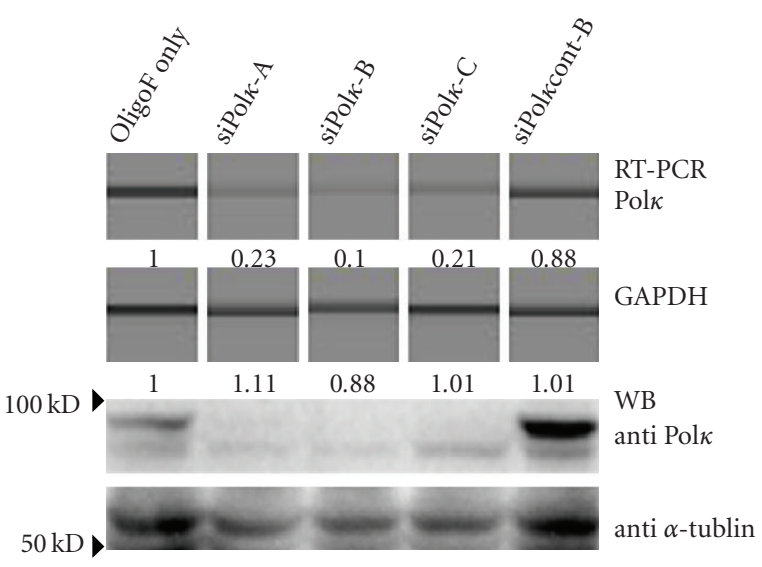

(a) RT-PCR and western blotting

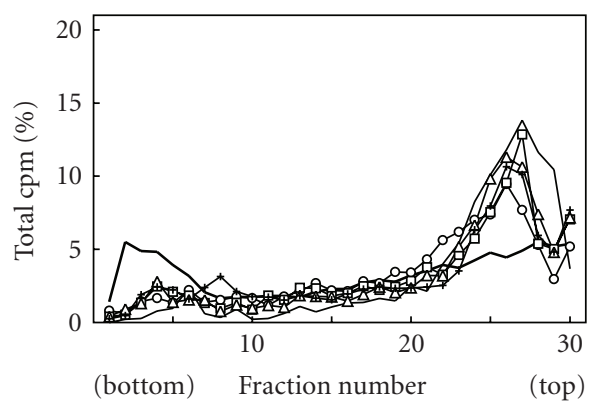

$\begin{array}{lc} & \text { Median }( \\ - \text { Pulse } & {[0.23]} \\ - \text { siPol } \kappa \text { cont-B }(100 \mathrm{nM}) & {[0.47]} \\ \multimap-\text { siPol } \kappa-\mathrm{A}(1 \mathrm{nM}) & {[0.32]} \\ \square-\text { siPol } \kappa-\mathrm{A}(5 \mathrm{nM}) & {[0.29]} \\ \square-\text { siPol } \kappa-\mathrm{A}(25 \mathrm{nM}) & {[0.26]} \\ \square-\text { siPol } \kappa-\mathrm{A}(100 \mathrm{nM}) & {[0.29]}\end{array}$

(c) Dose-response $($ siPol $\kappa$-A)

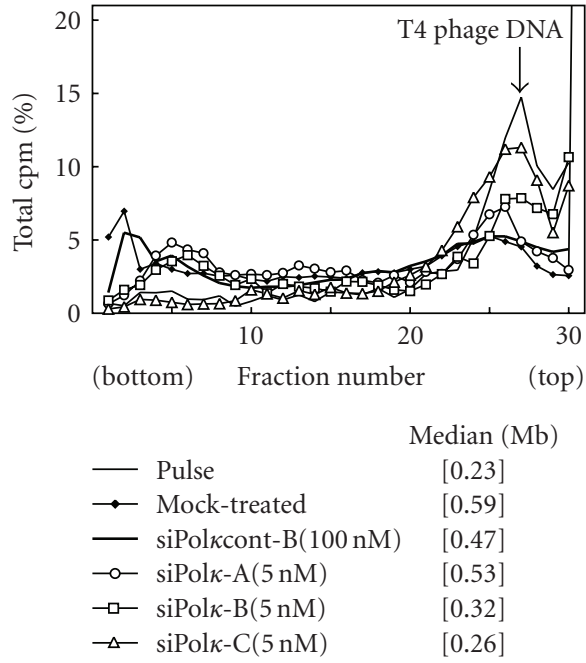

(b) Effects of various siRNAs (UV)
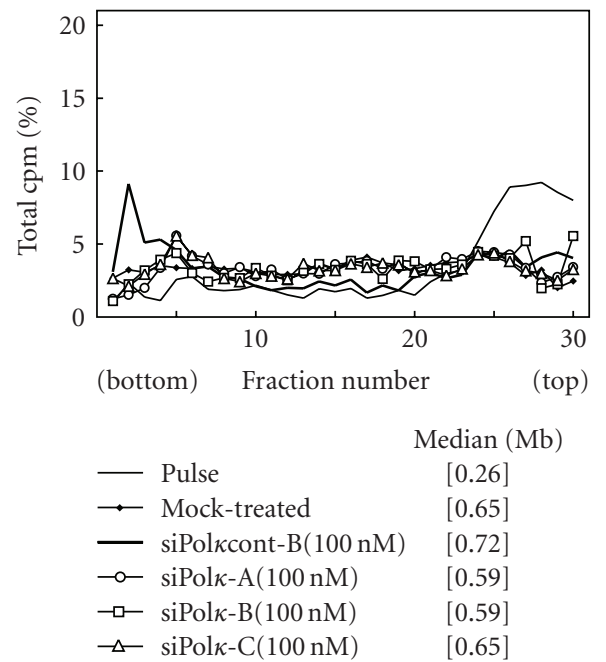

(d) Effect of various siRNAs (noUV)

FIGURE 6: Efficient knockdown by Pol $\kappa$ siRNAs and the effects on UV-induced TLS in HeLa cells (ASDG profiles of replication products). (a) Efficiency of knockdown on Pol $\kappa$ expression (RT-PCR analysis and western blot analysis), (b) Effects of various Pol $\kappa$ siRNAs on UVTLS, (c) Dose-response of siPol $\kappa$-A on UV-TLS, (d) Effect of various Pol $\kappa$ siRNAs (no UV control). Twenty-four hours after Pol $\kappa$ siRNA transfection, total RNA was isolated and Pol $\kappa$ RNA was quantified by RT-PCR. Results were shown in MultiNA gel images and the expression level was presented under the panel (a). Forty hours after Pol $\kappa$ siRNA transfection, whole cell extracts were prepared, and Pol $\kappa$ protein was quantified by western blot analysis (a). Forty hours after Pol $\kappa$ siRNA transfection, cells were UV-irradiated $\left(10 \mathrm{~J} / \mathrm{m}^{2}\right)$, incubated in normal medium for 30 minutes, pulse-labelled with $10 \mu \mathrm{Ci} / \mathrm{mL}$ of $\left[{ }^{14} \mathrm{C}\right]$ thymidine for 1 hour, then washed twice with PBS, and incubated for 5 hours at $37^{\circ} \mathrm{C}$ in normal medium (b, c). Forty hours after Pol $\kappa$ siRNA transfection, cells were not UV-irradiated, pulse-labelled with $10 \mu \mathrm{Ci} / \mathrm{mL}$ of $\left[{ }^{14} \mathrm{C}\right]$ thymidine for 30 minutes, washed twice with PBS, and incubated at $37^{\circ} \mathrm{C}$ in normal medium for 1 hour (d). Some of these profiles overlap (c, d). Sedimentation is from right to left. The arrow indicates the position of T4 phage DNA (166 kb, i.e., approximately $5.5 \times 10^{7} \mathrm{Da}$ /single strand). Average fragment length (in $\mathrm{Mb}$ ) of each profile is shown in square brackets.

possess high NER activity (unpublished observation), it is reasonable to conclude that our phenomena observed in HeLa cells by ASDG are largely attributable to CPD, although we have not yet determined the extent of remaining (6-4)PP.

We may also conclude that Rev1 is indispensable for TLS across CPD. Thus far, it is unknown if Rev1 is equally involved in TLS across CPD and (6-4)PP, or if it exhibits some preference. Nelson et al. [25] demonstrated that Revlp participates in UV-TLS across (6-4)PP, based on yeast transfected with a (6-4)PP-carrying plasmid; only slight differences were observed with a CPD-carrying plasmid.

In vitro lesion-bypass assay showed that $\mathrm{Pol} \eta$ alone accomplishes bypass across TT-CPD as above (i.e., both insertion and extension) [8, 9]. However, Yoon et al. 
presented complex results showing involvement of multiple bypass polymerases. They used SV-untransformed XP-A and XP-V cells, but did not include SV-untransformed normal fibroblasts, wherein we detected quick and caffeineinsensitive UV-TLS $[35,36]$. It is possible that the kind of damage, as well as cell status (normal, transformed, or cancerous) may determine the participation of bypass polymerase(s).

We have presented the first apparent evidence that Pol $\kappa$ participates in UV-TLS. Pol $\kappa$ knockout mouse embryonic cells are known to be UV sensitive [47], but the mechanism had not yet been determined. Pol $\kappa$ is also thought to play a part in the repair-synthesis step of NER [48, 49]. From the results of lesion-bypass assays, human Pol $\kappa$ was suggested to be unable to bypass CPD or (6-4)PP. Because the outcomes of such in vitro assays depend on the assay conditions [12], these results must be validated in vivo, such as by ASDG analysis.

\section{Conclusions}

Using siRNAs originally designed and ASDG technique, we verified the participation of multiple bypass polymerases in UV-induced TLS in HeLa cells, which is the consistent with recent model of 2 polymerase mechanisms. UV-TLS was largely abolished by siRNAs to Rev3 or Rev7, suggesting that these 2 proteins, which constitute Pol $\zeta$, play a primary role in mutagenic TLS. Rev1-targeted siRNAs also significantly abolished UV-TLS, consistent with prior suggestions that Rev1 is indispensable in mammalian mutagenic TLS. Unexpectedly, siRNAs to Pol $\eta$ prevented TLS to a great extent, implying that the Pol $\eta$ - and $\mathrm{Pol} \zeta$-dependent processes do not alternate but overlap. Pol $\kappa$ siRNAs, but not siRNAs to Pol $\iota$, delayed TLS; this is the first apparent evidence for the participation of Pol $\kappa$ in UV-TLS.

\section{Acknowledgments}

The authors are grateful to Dr. Hisao Masai (Tokyo Metropolitan Institute of Medical Science) for kind advice to siRNA design and transfection. This work was supported in part by Grants for Nuclear Research and Scientific Research from the Ministry of Education, Culture, Sports, Science, and Technology, Japan and by a Grant from the Mishima Kaiun Memorial Foundation. The author have no financial conflict of interests.

\section{References}

[1] A. R. Lehmann, A. Niimi, T. Ogi et al., "Translesion synthesis: Y-family polymerases and the polymerase switch," DNA Repair, vol. 6, no. 7, pp. 891-899, 2007.

[2] E. C. Friedberg, G. C. Walker, and W. Siede, DNA Repair and Mutagenesis, ASM Press, Washington, DC, USA, 1995.

[3] M. J. Horsfall, A. Borden, and C. W. Lawrence, "Mutagenic properties of the T-C cyclobutane dimer," Journal of Bacteriology, vol. 179, no. 9, pp. 2835-2839, 1997.

[4] D. L. Mitchell and R. S. Nairn, "The biology of the (6-4) photoproduct," Photochemistry and Photobiology, vol. 49, no. 6, pp. 805-819, 1989.
[5] S. Prakash and L. Prakash, "Translesion DNA synthesis in eukaryotes: a one- or two-polymerase affair," Genes and Development, vol. 16, no. 15, pp. 1872-1883, 2002.

[6] S. Prakash, R. E. Johnson, and L. Prakash, "Eukaryotic translesion synthesis DNA polymerases: specificity of structure and function," Annual Review of Biochemistry, vol. 74, pp. 317-353, 2005.

[7] J. E. Cleaver and K. H. Kraemer, "Xeroderma pigmentosum and cockayne syndrome," in The Metabolic and Molecular Basis of Inherited Disease, C. R. Scriver, A. L. Beaudet, W. S. Sly, and D. Valle, Eds., vol. 3, pp. 4393-4419, McGraw-Hill, New York, NY, USA, 7th edition, 1995.

[8] C. Masutani, R. Kusumoto, S. Iwai, and F. Hanaoka, "Mechanisms of accurate translesion synthesis by human DNA polymerase $\eta$," EMBO Journal, vol. 19, no. 12, pp. 3100-3109, 2000.

[9] C. Masutani, R. Kusumoto, A. Yamada et al., "Xeroderma pigmentosum variant: from a human genetic disorder to a novel DNA polymerase," Cold Spring Harbor Symposia on Quantitative Biology, vol. 65, pp. 71-80, 2000.

[10] S. Prakash, R. E. Johnson, M. T. Washington, L. Haracska, C. M. Kondratick, and L. Prakash, "Role of yeast and human DNA polymerase $\eta$ in error-free replication of damaged DNA," Cold Spring Harbor Symposia on Quantitative Biology, vol. 65 , pp. 51-59, 2000.

[11] C. Masutani, R. Kusumoto, A. Yamada et al., "The XPV (xeroderma pigmentosum variant) gene encodes human DNA polymerase $\eta$," Nature, vol. 399, no. 6737, pp. 700-704, 1999.

[12] J. P. McDonald, V. Rapić-Otrin, J. A. Epstein et al., "Novel human and mouse homologs of Saccharomyces cerevisiae DNA polymerase $\eta$," Genomics, vol. 60, no. 1, pp. 20-30, 1999.

[13] A. Tissier, E. G. Frank, J. P. McDonald, S. Iwai, F. Hanaoka, and R. Woodgate, "Misinsertion and bypass of thymine-thymine dimers by human DNA polymerase ı," EMBO Journal, vol. 19, no. 19 , pp. 5259-5266, 2000.

[14] V. L. Gerlach, W. J. Feaver, P. L. Fischhaber et al., "Human DNA polymerase $\kappa$ : a novel DNA polymerase of unknown biological function encoded by the DINB1 gene," Cold Spring Harbor Symposia on Quantitative Biology, vol. 65, pp. 41-49, 2000.

[15] E. Ohashi, T. Ogi, R. Kusumoto et al., "Error-prone bypass of certain DNA lesions by the human DNA polymerase $\kappa$," Genes and Development, vol. 14, no. 13, pp. 1589-1594, 2000.

[16] E. Ohashi, K. Bebenek, T. Matsuda et al., "Fidelity and processivity of DNA synthesis by DNA polymerase $\kappa$, the product of the human DINB1 gene," Journal of Biological Chemistry, vol. 275, no. 50, pp. 39678-39684, 2000.

[17] C. W. Lawrence and R. B. Christensen, "Ultraviolet-induced reversion of $c y c 1$ alleles in radiation-sensitive strains of yeast. III. rev3 mutant strains," Genetics, vol. 92, no. 2, pp. 397-408, 1979.

[18] P. E. M. Gibbs, X.-D. Wang, Z. Li et al., "The function of the human homolog of Saccharomyces cerevisiae REV1 is required for mutagenesis induced by UV light," Proceedings of the National Academy of Sciences of the United States of America, vol. 97, no. 8, pp. 4186-4191, 2000.

[19] P. E. M. Gibbs, W. G. McGregor, V. M. Maher, P. Nisson, and C. W. Lawrence, "A human homolog of the Saccharomyces cerevisiae REV3 gene, which encodes the catalytic subunit of DNA polymerase $\zeta$," Proceedings of the National Academy of Sciences of the United States of America, vol. 95, no. 12, pp. 6876-6880, 1998.

[20] Y. Murakumo, T. Roth, H. Ishii et al., "A human REV7 homolog that interacts with the polymerase $\zeta$ catalytic subunit 
hREV3 and the spindle assembly checkpoint protein hMAD2," Journal of Biological Chemistry, vol. 275, no. 6, pp. 4391-4397, 2000.

[21] C. W. Lawrence, "Cellular roles of DNA polymerase $\zeta$ and Rev1 protein," DNA Repair, vol. 1, no. 6, pp. 425-435, 2002.

[22] Z. Li, H. Zhang, T. P. McManus, J. J. McCormick, C. W. Lawrence, and V. M. Maher, "hREV3 is essential for error-prone translesion synthesis past UV or benzo[a]pyrene diol epoxide-induced DNA lesions in human fibroblasts," Mutation Research, vol. 510, no. 1-2, pp. 71-80, 2002.

[23] K. McNally, J. A. Neal, T. P. McManus, J. J. McCormick, and V. M. Maher, "hRev7, putative subunit of $\mathrm{hPol} \zeta$, plays a critical role in survival, induction of mutations, and progression through S-phase, of UV(254 nm)-irradiated human fibroblasts," DNA Repair, vol. 7, no. 4, pp. 597-604, 2008.

[24] M. Diaz, N. B. Watson, G. Turkington, L. K. Verkoczy, N. R. Klinman, and W. G. McGregor, "Decreased frequency and highly aberrant spectrum of ultraviolet-induced mutations in the hprt gene of mouse fibroblasts expressing antisense RNA to DNA polymerase $\zeta$," Molecular Cancer Research, vol. 1, no. 11, pp. 836-847, 2003.

[25] J. R. Nelson, P. E. M. Gibbs, A. M. Nowicka, D. C. Hinkle, and C. W. Lawrence, "Evidence for a second function for Saccharomyces cerevisiae Rev1p," Molecular Microbiology, vol. 37, no. 3, pp. 549-554, 2000.

[26] C. Guo, T.-S. Tang, M. Bienko et al., "Ubiquitin-binding motifs in REV1 protein are required for its role in the tolerance of DNA damage," Molecular and Cellular Biology, vol. 26, no. 23, pp. 8892-8900, 2006.

[27] C. Guo, P. L. Fischhaber, M. J. Luk-Paszyc et al., "Mouse Rev1 protein interacts with multiple DNA polymerases involved in translesion DNA synthesis," EMBO Journal, vol. 22, no. 24, pp. 6621-6630, 2003.

[28] P. L. Kannouche, J. Wing, and A. R. Lehmann, "Interaction of human DNA polymerase $\eta$ with monoubiquitinated PCNA: a possible mechanism for the polymerase switch in response to DNA damage," Molecular Cell, vol. 14, no. 4, pp. 491-500, 2004.

[29] K. Watanabe, S. Tateishi, M. Kawasuji, T. Tsurimoto, H. Inoue, and M. Yamaizumi, "Rad18 guides pol $\eta$ to replication stalling sites through physical interaction and PCNA monoubiquitination," EMBO Journal, vol. 23, no. 19, pp. 3886-3896, 2004.

[30] P. L. Fischhaber and E. C. Friedberg, "How are specialized (low-fidelity) eukaryotic polymerases selected and switched with high-fidelity polymerases during translesion DNA synthesis?" DNA Repair, vol. 4, no. 2, pp. 279-283, 2005.

[31] A. R. Lehmann, S. Kirk Bell, C. F. Arlett et al., "Xeroderma pigmentosum cells with normal levels of excision repair have a defect in DNA synthesis after UV irradiation," Proceedings of the National Academy of Sciences of the United States of America, vol. 72, no. 1, pp. 219-223, 1975.

[32] A. R. Lehmann, "Postreplication repair of DNA in ultravioletirradiated mammalian cells," Journal of Molecular Biology, vol. 66, no. 3, pp. 319-337, 1972.

[33] A. R. Lehmann, "Postreplication repair of DNA in mammalian cells," Life Sciences, vol. 15, no. 12, pp. 2005-2016, 1974.

[34] K. Yamada, Y. Kameyama, and S. Inoue, "An improved method of alkaline sucrose density gradient sedimentation to detect less than one lesion per $1 \mathrm{Mb}$ DNA," Mutation Research, vol. 364, no. 2, pp. 125-131, 1996.

[35] K. Yamada, J. Takezawa, and O. Ezaki, “Translesion replication in cisplatin-treated xeroderma pigmentosum variant cells is also caffeine-sensitive: features of the error-prone DNA polymerase(s) involved in UV-mutagenesis," DNA Repair, vol. 2, no. 8, pp. 909-924, 2003.

[36] J. Takezawa, Y. Ishimi, and K. Yamada, "Proteasome inhibitors remarkably prevent translesion replication in cancer cells but not normal cells," Cancer Science, vol. 99, no. 5, pp. 863-871, 2008.

[37] J.-H. Choi and G. P. Pfeifer, "The role of DNA polymerase $\eta$ in UV mutational spectra," DNA Repair, vol. 4, no. 2, pp. 211220, 2005.

[38] J.-H. Choi, A. Besaratinia, D.-H. Lee, C.-S. Lee, and G. P. Pfeifer, "The role of DNA polymerase $\iota$ in UV mutational spectra," Mutation Research, vol. 599, no. 1-2, pp. 58-65, 2006.

[39] K. Machida, K. T.-N. Cheng, V. M.-H. Sung et al., "Hepatitis $\mathrm{C}$ virus induces a mutator phenotype: enhanced mutations of immunoglobulin and protooncogenes," Proceedings of the National Academy of Sciences of the United States of America, vol. 101, no. 12, pp. 4262-4267, 2004.

[40] S. Shachar, O. Ziv, S. Avkin et al., "Two-polymerase mechanisms dictate error-free and error-prone translesion DNA synthesis in mammals," EMBO Journal, vol. 28, no. 4, pp. 383393, 2009.

[41] J. K. Hicks, C. L. Chute, M. T. Paulsen et al., "Differential roles for DNA polymerases eta, zeta, and REV1 in lesion bypass of intrastrand versus interstrand DNA cross-links," Molecular and Cellular Biology, vol. 30, no. 5, pp. 1217-1230, 2010.

[42] C. E. Edmunds, L. J. Simpson, and J. E. Sale, "PCNA ubiquitination and REV1 define temporally distinct mechanisms for controlling translesion synthesis in the avian cell line DT40," Molecular Cell, vol. 30, no. 4, pp. 519-529, 2008.

[43] T. Matsuda, K. Bebenek, C. Masutani, F. Hanaoka, and T. A. Kunkel, "Low fidelity DNA synthesis by human DNA polymerase- $\eta$," Nature, vol. 404, no. 6781, pp. 1011-1013, 2000.

[44] K. Bebenek, T. Matsuda, C. Masutani, F. Hanaoka, and T. A. Kunkel, "Proofreading of DNA polymerase $\eta$-dependent replication errors," Journal of Biological Chemistry, vol. 276, no. 4, pp. 2317-2320, 2001.

[45] J.-H. Yoon, L. Prakash, and S. Prakash, "Highly error-free role of DNA polymerase $\eta$ in the replicative bypass of UV-induced pyrimidine dimers in mouse and human cells," Proceedings of the National Academy of Sciences of the United States of America, vol. 106, no. 43, pp. 18219-18224, 2009.

[46] J.-H. Yoon, L. Prakash, and S. Prakash, "Error-free replicative bypass of $(6-4)$ photoproducts by DNA polymerase $\zeta$ in mouse and human cells," Genes and Development, vol. 24, no. 2, pp. 123-128, 2010.

[47] T. Ogi, Y. Shinkai, K. Tanaka, and H. Ohmori, "Pol $\kappa$ protects mammalian cells against the lethal and mutagenic effects of benzo[a]pyrene," Proceedings of the National Academy of Sciences of the United States of America, vol. 99, no. 24, pp. 15548-15553, 2002.

[48] T. Ogi and A. R. Lehmann, "The Y-family DNA polymerase $\kappa$ (pol $\kappa$ ) functions in mammalian nucleotide-excision repair," Nature Cell Biology, vol. 8, no. 6, pp. 640-642, 2006.

[49] T. Ogi, S. Limsirichaikul, R. M. Overmeer et al., "Three DNA polymerases, recruited by different mechanisms, carry out NER repair synthesis in human cells," Molecular Cell, vol. 37, no. 5, pp. 714-727, 2010. 

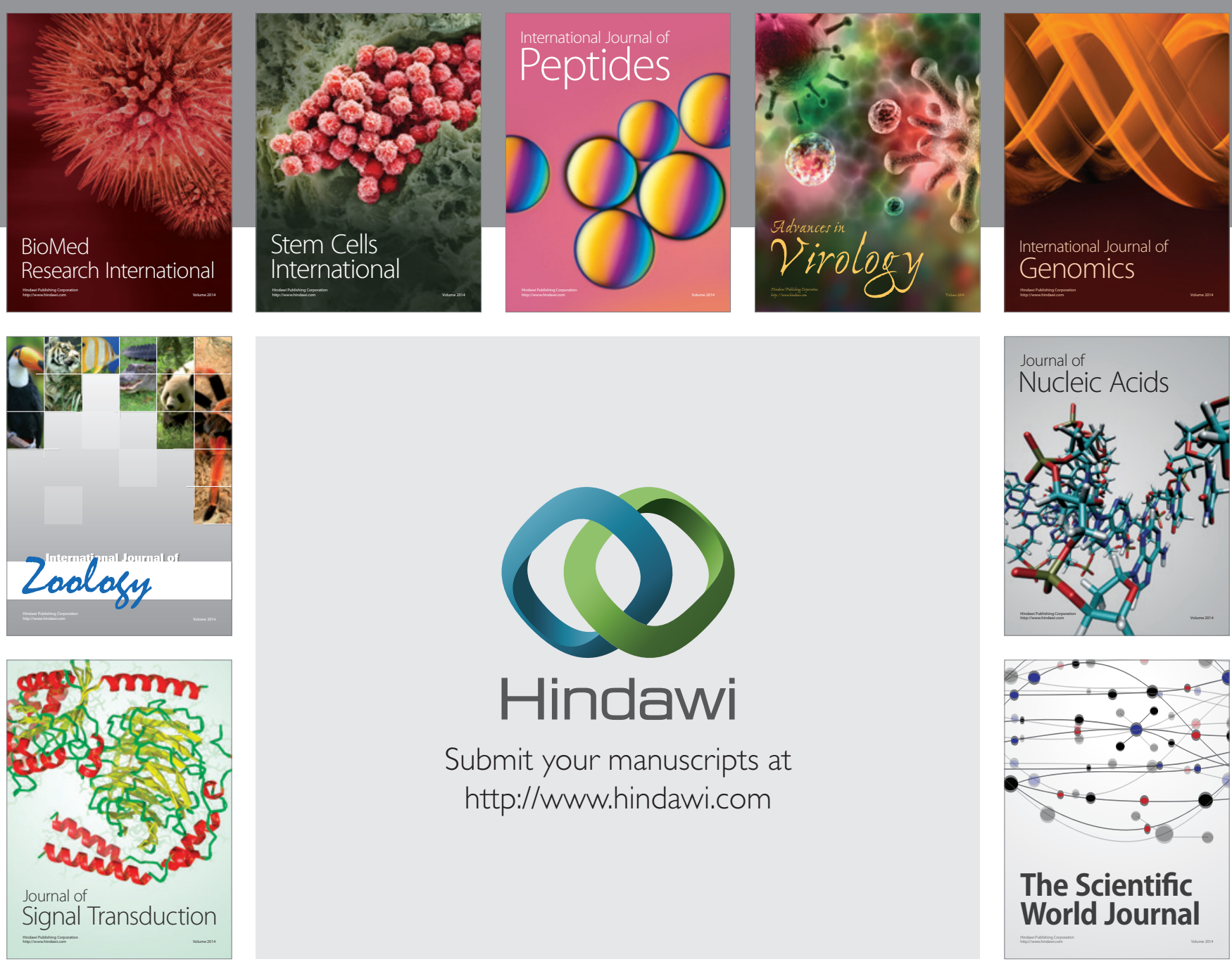

Submit your manuscripts at

http://www.hindawi.com
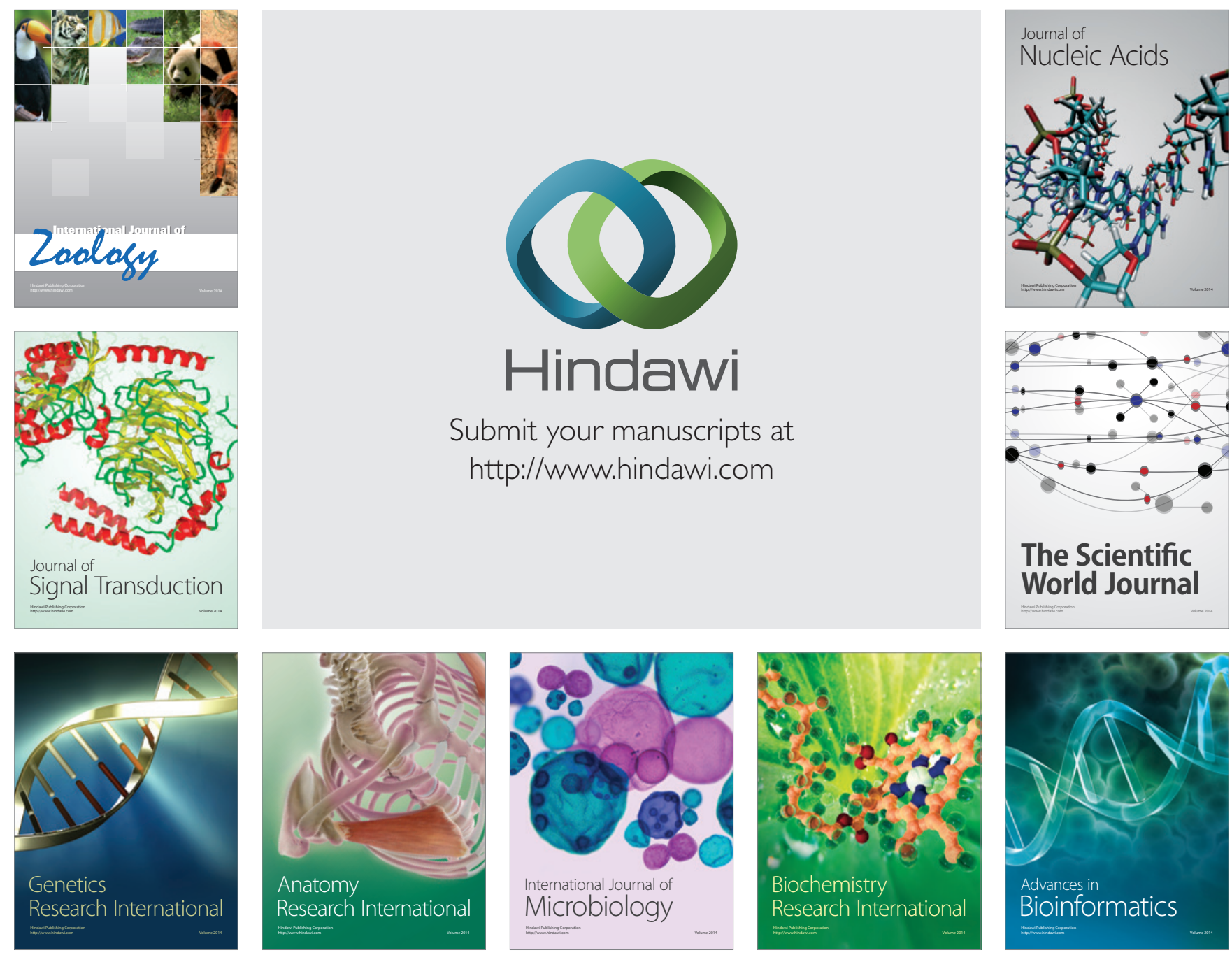

The Scientific World Journal
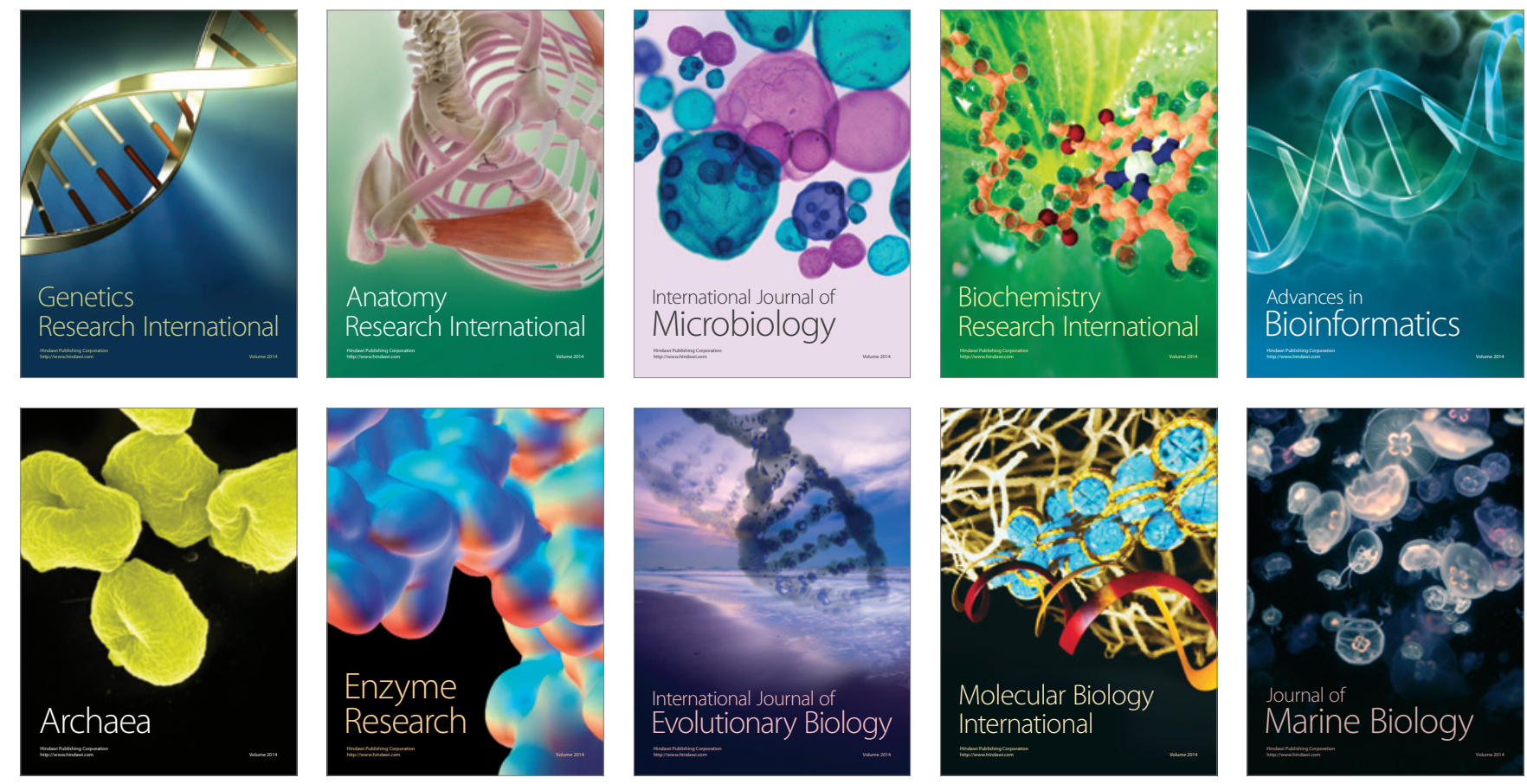